\title{
ANALYSIS OF THE RISK PREMIUM IN THE FORWARD MARKET FOR SALMON
}

\author{
FRED ESPEN BENTH, ANNE MARIA EIKESET, SIMON ASHER LEVIN AND WANJUAN REN
}

\begin{abstract}
We analyse forward prices observed at the Fishpool market, and propose a two-factor continuoustime stochastic process for modelling the time dynamics. The data analysis reveals that the two factors can be assumed to be a non-stationary compound Poisson process and a stationary continuous-time autoregressive dynamics, describing the bumps observed in the forward curves. We use the model to analyse the risk premium in the forward markets, and find a negative premium in the long end of the market which is in line with the theory of normal backwardation. However, contracts with short time to maturity have a risk premium with randomly changing sign, pointing towards a hedging pressure also induced by the demand-side of the market.
\end{abstract}

\section{INTRODUCTION}

In this paper we analyse the risk premium in the forward market for salmon using stochastic modelling and pricing techniques from financial mathematics. At the Fish Pool $^{1}$ market one can trade in forward contracts with delivery of salmon. The contracts are financially settled against the weekly announced Fish Pool Index (FPI), over a monthly settlement period. This means that a long position in a forward contract will give the value of the FPI over the settlement period in return for the agreed forward price.

One of our main motivations for conducting this study is to understand whether futures may be affected by increased volatility in fish stocks, the commodity, and environmental variation such as climate change. To use fish futures for marine conservation was suggested by Sugihara in the early 2000s (Dalton [10]) and more recently the price dynamics of such futures contracts have been studied (Boston [8], Asche et al. [1]). However, Asche et al. [1] did not find a change in the volatility between species or between farmed and wild salmon, suggesting they had a small impact on price volatility due to their limited trade volumes. The FPI data have also been investigated before in related models (Ewald [13], Ewald \& Salehi[14]); however, they have used different models with different focus than ours.

Financial theory claims that there is a risk premium in financial markets. In a stylized way, risk is paid by expected return, so that higher risk comes with an increased expected return. We want to use this as a background for exploring a possible biological risk premium in the markets, specifically, how futures on fish products are affected by increased volatility in the fish stocks (the commodity), and environmental variation (such as climate change). The only financial instrument that exists in the world on marine resources involves futures contracts on the Norwegian Fish Pool Index (FPI, http://fishpool.eu/), which reflects the spot price for fish products, mainly fresh, farmed Atlantic salmon (Salmo salar).

Typically in commodity markets, a forward contract is settled with delivery of the commodity at a given time, the so-called delivery time. The forward price is derived by predicting the spot price of the commodity at delivery, based on a risk adjustment, and will become an explicit function of the current spot price. This risk adjustment includes storage costs, liquidity premium, convenience yield and other market frictions. We refer to Geman [16] for a discussion of various commodity forward markets. In the case of the Fish Pool forwards, the "spot" is the FPI, with the peculiar feature that forwards can be traded in the market daily, whereas the "spot" is an index only announced every Tuesday. Hence, in their trading operation, actors in fish contracts must base their forward prices on predictions of the FPI except on Tuesdays when it is revealed.

Date: Monday $7^{\text {th }}$ August, 2017.

Key words and phrases. Salmon futures, autoregressive models in continuous time, futures prices.

The preparation of this paper has been supported by the Nordforsk-funded project Green Growth Based on Marine Resources: Ecological and Socio-Economic Constraints (GreenMAR) and by NSF grants GEO-1211972 and OCE-1246746. We are grateful to Simen Thorbeck, Head of Trading at fishpool.eu, and Sigbjørn Tveterås at University of Stavanger, Norway (UiS) for discussions on market and data. The authors are listed in alphabetical order.

${ }^{1}$ See www.fishpool.eu 

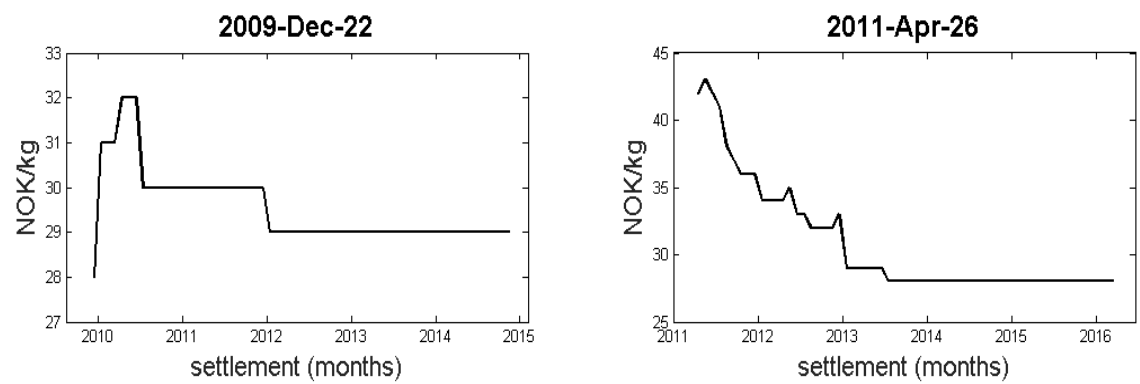

2013-Aug-12
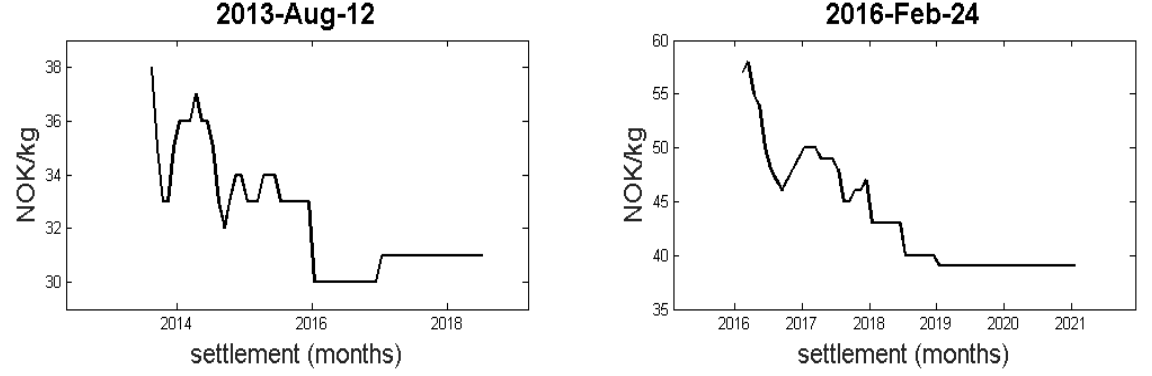

FIGURE 1. Plot of some example forward curves.

The Fish Pool Index (FPI) has been derived to provide a reference price reflecting the actual spot price of fresh Atlantic Salmon and is the basis for settlement of all financial salmon contracts at Fish Pool. It is based on three indices; the Nasdaq Salmon Index (Exporters selling prices), Fish Pool European Buyers Index (Large purchasers purchase price) and Statistics Norway customs statistics (SSB; Norwegian export statistics, ssb.no), and calculated as a monthly settlement price based on the 4-5 weekly price elements. All financial contracts at Fish Pool are settled on a monthly basis against FPI, which is published weekly on fishpool.eu.

To follow the framework for no-arbitrage pricing of forward contracts in commodity markets like energy, weather, precious metals etc.. (see Geman [16], Benth et al. [3], Benth and Šaltytė Benth [2]), we will in this paper develop a forward price dynamics based on a continuous-time stochastic process. Thus, we will assume that the market observes the FPI continuously over time. This is a bold approximation over reality, but as we will see, our approach will provide us with a term structure dynamics reflecting the qualitative behaviour of the observed market prices rather closely. In Figure 1 we show four different forward curves observed over the years in the Fish Pool market. Each of the curves consist of 60 forward prices at a given date, and we clearly see that in the long end of the market, prices are constant as a function of the settlement time. On the other hand, in the shorter end of the market, the term structure of the forward prices depict a much more complex behaviour with bumps in the very short end and also farther out on the curve. Over time, the forward curves change in shape and also show a variation in the long end prices. Motivated by these shapes, we propose a two-factor stochastic dynamics for the FPI, based on a non-stationary part and a continuous-time autoregressive moving average dynamics for the stationary part. Although the market only observes the FPI on Tuesdays, we propose a model being continuous over time accounting for the possibility that traders can make qualified assessments on the "current FPI". Using this model, we derive analytical forward prices from conditional risk-adjusted expected values of the FPI over the settlement period. We remark that our model for the FPI, although being classified as a two-factor model analogous to Gibson and Schwartz [17], is novel in the sense that we find a non-stationary compound Poisson process as the long-term factor, combined with a higher-order autoregressive short term factor.

From an empirical analysis based on a history of observed FPI data along with a long record of historical Fish Pool forward prices, we calibrate our model. The non-stationary FPI factor will describe the long end variations of the forward curve, while the continuous-time autoregressive dynamics will be sufficiently flexible in order to account for the complex shapes like those we see in Figure 1. We apply our stochastic model to analyse the risk premium in the Fish Pool market, defined as the difference between the forward 
prices and the predicted FPI over the corresponding settlement periods. We find that the risk premium in the long end of the market is negative, consistent with the normal backwardation theory of producers putting on a hedging pressure. However, in the shorter end of the market we find a risk premium with a randomly changing sign, indicating that also the demand-side of the fish market puts on a significant hedging pressure. This interesting feature of the fish market is shared with other "exotic" forward markets like power (see Benth, Cartea and Kiesel [4]). Geman and Vasicek [18] argues that the retailers in power market use forwards as a protection against sudden high prices. Indeed, their argument holds for nonstorable commodities. Our results indicate a similar situation for the Fish Pool forward market.

Our results are presented as follows: In Section 2 we present the stochastic model for the FPI along with a derivation of theoretical forward prices. Some analysis of the model is also performed, useful for the empirical analysis of FPI and Fish Pool forward contracts presented in Section 3. The next section contains an empirical study of the risk premium, before we conclude in the last section.

\section{A CONTINUOUS-TIME FORWARD PRICE MODEL}

In this section we define a spot dynamics for the FPI generalizing the two-factor model of Lucia and Schwartz [20], where the stationary part is modelled using a higher-order continuous-time autoregressive process. From the FPI dynamics we derive forward prices for the salmon market.

Let $(\Omega, \mathcal{F}, P)$ define a complete probability space, equipped with a filtration $\left\{\mathcal{F}_{t}\right\}_{t \geq 0}$. Recall from Brockwell [9] that a real-valued stochastic process $Y$ is called a continuous-time autoregressive process of order $p$ for $p \in \mathbb{N}$, if $Y(t)=\mathbf{e}_{1}^{\top} \mathbf{Z}(t)$ where $\mathbf{Z} \in \mathbb{R}^{p}$ is the vector-valued Ornstein-Uhlenbeck process given by

$$
d \mathbf{Z}(t)=A(\mathbf{Z}(t)-\boldsymbol{\xi}) d t+\sigma \mathbf{e}_{p} d B(t), \quad \mathbf{Z}(0)=\mathbf{Z}_{0} \in \mathbb{R}^{p},
$$

for a Brownian motion $B$. Here, $\left\{\mathbf{e}_{k}\right\}_{k=1}^{p} \subset \mathbb{R}^{p}$ is the canonical basis of $\mathbb{R}^{p}$, while $\boldsymbol{\xi} \in \mathbb{R}^{p}$ and $\sigma>0$ are constants. The matrix $A \in \mathbb{R}^{p \times p}$ is defined as

$$
A=\left[\begin{array}{cccccc}
0 & 1 & 0 & 0 & \ldots & 0 \\
0 & 0 & 1 & 0 & \ldots & 0 \\
\cdot & \cdot & \cdot & . & \ldots & . \\
. & . & . & . & \ldots & . \\
0 & 0 & 0 & 0 & \ldots & 1 \\
-\alpha_{p} & -\alpha_{p-1} & -\alpha_{p-2} & -\alpha_{p-3} & \ldots & -\alpha_{1}
\end{array}\right]
$$

for positive constants $\alpha_{k}, k=1, \ldots, p$. Note that we have introduced a level $\boldsymbol{\xi}$ into the dynamics of $\mathbf{Z}$.

Lemma 1. For any $s \geq t \geq 0$, it holds

$$
\mathbf{Z}(s)=e^{A(s-t)} \mathbf{Z}(t)+\left(I-e^{A(s-t)}\right) \boldsymbol{\xi}+\sigma \int_{t}^{s} e^{A(s-u)} \mathbf{e}_{p} d B(u),
$$

where I denotes the $p \times p$-dimensional identity matrix.

Proof. The result follows directly by an application of the multidimensional Itô formula.

From this result we see that for any $s \geq t \geq 0$,

$$
Y(t)=\mathbf{e}_{1}^{\top} e^{A(s-t)} \mathbf{Z}(t)+\mathbf{e}_{1}^{\top}\left(I-e^{A(s-t)}\right) \boldsymbol{\xi}+\sigma \int_{t}^{s} \mathbf{e}_{1}^{\top} e^{A(s-u)} \mathbf{e}_{p} d B(u) .
$$

If the matrix $A$ has eigenvalues with negative real part, then $\mathbf{Z}$ admits a limiting distribution as $t \rightarrow \infty$. Indeed, $\mathbf{Z}(t)$ is $p$-variate Gaussian distributed, with limiting distribution also being $p$-variate Gaussian with mean

$$
\lim _{t \rightarrow \infty} \mathbb{E}[\mathbf{Z}(t)]=-\boldsymbol{\xi}
$$

and variance

$$
\lim _{t \rightarrow \infty} \operatorname{Var}(\mathbf{Z}(t))=\sigma^{2} \int_{0}^{\infty} e^{A u} \mathbf{e}_{p} \mathbf{e}_{p}^{\top} e^{A^{\top} u} d u
$$

We will assume that $A$ has eigenvalues with negative real part in this paper, which implies that $Y$ has a limiting distribution. In this sense we may refer to $Y$ as a stationary process. 
From now on we refer to the process $Y$ as a CAR $(p)$-process as $Y$ can be intepreted as a continuous-time version of a autoregressive process of order $p$ (see Brockwell [9]).

Let the FPI dynamics at time $t \geq 0$ be denoted by $S(t)$, and assume that

$$
S(t)=\exp (X(t)+Y(t))
$$

where $X(t)=L(t)$, a square-integrable Lévy process. We assume that $L$ has finite exponential moments of all orders. Typically, in the context of Lucia and Schwartz [20],

$$
L(t)=\mu d t+\eta d W(t)
$$

for a Brownian motion $W$ and $\mu, \eta>0$ being two constants. In our analysis here we will assume that $L$ is independent of $B$, but remark that for the Lucia and Schwartz model the two Brownian motions are correlated. However, as our empirical analysis will show, the Lévy process can be conveniently modelled as a compound Poisson process, which is a pure-jump process and thus must be independent of a continuoustime Brownian motion $B$. We see that the logarithmic FPI dynamics follows a non-stationary process $X$, being a Lévy process, and a stationary process $Y$, and thus is an extension of the two-factor model considered by Lucia and Schwartz [20]. The non-stationary process $X$ is the long-term factor, while $Y$ is the short-term fluctuations of the FPI.

We next derive the forward price dynamics of contracts settled on the FPI. To this end, let $f(t, T)$ denote the forward price at time $t \leq T$, for a contract with settlement at time $T \geq 0$, and following Duffie [12] we define

$$
f(t, T)=\mathbb{E}_{Q}\left[S(T) \mid \mathcal{F}_{t}\right]
$$

where $Q \sim P$ is a pricing measure. In order to calculate this expectation, we need to know the dynamics of $S$ with respect to the probability measure $Q$, which we discuss next.

From Girsanov's Theorem (see Karatzas and Shreve [19]) and the Esscher transform (see Benth et al. [3]), for two constants $\theta_{B}, \theta_{L}$ there exists a probability $Q \sim P$ such that $\widetilde{B}$

$$
d \widetilde{B}=\theta_{B} d t+d B(t)
$$

is a Brownian motion with respect to $Q$ and $L$ is a Lévy process with cumulant $\psi_{Q}(x)=\psi\left(x-\mathrm{i} \theta_{L}\right)-$ $\psi\left(-\mathrm{i} \theta_{L}\right)$, independent from $\widetilde{B}$. Here, $\psi$ is the cumulant of $L$, that is the logarithm of the characteristic function of $L(1)$. The constants $\theta_{B}$ and $\theta_{L}$ parametrize the risk premium in the market, and we will refer to them as the market price of risk factors. Indeed, $\theta_{L}$ is the long-term market price of risk, while $\theta_{B}$ is the short-term market price of risk. The $Q$-dynamics of $\mathbf{Z}$ becomes,

$$
d \mathbf{Z}(t)=A\left(\mathbf{Z}(t)-\boldsymbol{\xi}-\theta_{B} \mathbf{e}_{p}\right) d t+\sigma \mathbf{e}_{p} d \widetilde{B}(t)
$$

We find:

Proposition 2. For any $T \geq t \geq 0$, the forward price $f(t, T)$ is

$$
f(t, T)=\exp \left(h(T-t)+X(t)+\mathbf{e}_{1}^{\top} e^{A(T-t)} \mathbf{Z}(t)\right)
$$

where

$$
h(x)=\psi_{Q}(-\mathrm{i}) x+\mathbf{e}_{1}^{\top}\left(I-e^{A x}\right)\left(\boldsymbol{\xi}+\theta_{B} \mathbf{e}_{p}\right)+\frac{1}{2} \int_{0}^{x} \sigma^{2}\left(\mathbf{e}_{1}^{\top} e^{A u} \mathbf{e}_{p}\right)^{2} d u,
$$

for $x \geq 0$.

Proof. Similarly to Lemma 1, we have from (7)

$$
\mathbf{Z}(T)=e^{A(T-t)} \mathbf{Z}(t)+\left(I-e^{A(T-t)}\right)\left(\boldsymbol{\xi}+\theta_{B} \mathbf{e}_{p}\right)+\sigma \int_{t}^{T} e^{A(T-u)} \mathbf{e}_{p} d \widetilde{B}(u)
$$


for $T \geq t$. Using the fact that $\widetilde{B}$ is a Brownian motion and $L$ is a Lévy process under $Q$, being independent, we find from the $\mathcal{F}_{t}$-measurability of $\mathbf{Z}(t)$ and $X(t)$ that

$$
\begin{aligned}
& f(t, T)= \mathbb{E}_{Q}\left[\exp (X(T)+Y(T)) \mid \mathcal{F}_{t}\right] \\
&=\exp \left(X(t)+\mathbf{e}_{1}^{\top} e^{A(T-t)} \mathbf{Z}(t)+\mathbf{e}_{1}^{\top}\left(I-e^{A(T-t)}\right)\left(\boldsymbol{\xi}+\theta_{B} \mathbf{e}_{p}\right)\right) \\
&\left.\quad \times \mathbb{E}_{Q}\left[\exp \left(\sigma \int_{t}^{T} \mathbf{e}_{1}^{\top} e^{A(T-u)} \mathbf{e}_{p} d \widetilde{B}(u)\right)\right] \times \mathbb{E}_{Q}[\exp (L(T)-L(t)))\right] .
\end{aligned}
$$

Hence, the result follows after a calculation of the last expectation using standard properties of the normal distribution and the cumulant of $L$ under $Q$.

The long end of the forward curve is controlled by the long term factor, as the next result shows:

Proposition 3. If $p>1$ it holds that

$$
\lim _{T \rightarrow \infty}\left(\ln f(t, T)-\psi_{Q}(-\mathrm{i})(T-t)\right)=X(t)+c
$$

for any $t \geq 0$, where

$$
c=\mathbf{e}_{1}^{\top} \boldsymbol{\xi}+\frac{1}{2} \int_{0}^{\infty} \sigma^{2}\left(\mathbf{e}_{1}^{\top} e^{A u} \mathbf{e}_{p}\right)^{2} d u
$$

Proof. From Prop. 2 we find

$$
\ln f(t, T)-\psi_{Q}(-\mathrm{i})(T-t)=X(t)+\mathbf{e}_{1}^{\top} e^{A(T-t)} \mathbf{Z}(t)+c(T-t)
$$

for

$$
c(x):=\mathbf{e}_{1}^{\top}\left(I-e^{A x}\right)\left(\boldsymbol{\xi}+\theta_{B} \mathbf{e}_{p}\right)+\frac{1}{2} \int_{0}^{x} \sigma^{2}\left(\mathbf{e}_{1}^{\top} e^{A u} \mathbf{e}_{p}\right)^{2} d u
$$

for $x \geq 0$. Since, by assumption, the eigenvalues of $A$ have negative real part, $\lim _{T \rightarrow \infty} \mathbf{e}_{1}^{\top} e^{A(T-t)} \mathbf{Z}(t)=$ 0 . Furthermore, since $\mathbf{e}_{1}^{\top} \mathbf{e}_{p}=0$, we find $\lim _{x \rightarrow \infty} c(x)=c$ with $c$ defined in the Proposition. The result follows.

In the case $p=1$, the CAR(1)-process $Y$ will be a classical Ornstein-Uhlenbeck process. In this situation the constant $c$ in Prop. 3 becomes

$$
c=\xi+\theta_{B}+\frac{1}{2} \int_{0}^{\infty} \sigma^{2} e^{-2 \alpha_{1} u} d u=\xi+\theta_{B}+\frac{\sigma^{2}}{4 \alpha_{1}},
$$

since $\mathbf{e}_{p}=\mathbf{e}_{1}=1$ and $A=-\alpha_{1}<0$. Note that we have used the notation $\xi$ for $\boldsymbol{\xi} \in \mathbb{R}$.

What we see from Prop. 3 (and also the special case $p=1$ ) is that the dynamics of $f(t, T)$ for $T$ large is essentially given by $\exp (X(t))$. In other words, the long term factor can be traded, at least approximately, since it can be viewed as a forward contract with $T>t$. By constructing a roll-over portfolio in the forward contract with longest time until maturity, we essentially have a position in the long term factor $X$. By no-arbitrage principles, $t \mapsto \exp (X(t))$ must therefore be a martingale under $Q$. Hence, we specify $\theta_{L}$ to be such that $\mathbb{E}_{Q}[\exp (L(t))]=1$, which is equivalent to

$$
\psi_{Q}(-\mathrm{i})=0 \text {. }
$$

From now on, we will stick to this choice of $\theta_{L}$.

We recall that in the Fish Pool market does not trade in contracts with fixed maturity time $T$, but with a settlement period $\left[T_{1}, T_{2}\right]$. The forward contracts are settled on the average FPI over $\left[T_{1}, T_{2}\right]$, and thus we have

$$
F\left(t, T_{1}, T_{2}\right)=\frac{1}{T_{2}-T_{1}} \int_{T_{1}}^{T_{2}} f(t, T) d T .
$$

We notice from Prop. 3 that when $T_{1}>>t, f(t, T) \approx \exp (c+X(t))$, and therefore

$$
F\left(t, T_{1}, T_{2}\right) \approx \exp (c+X(t)) .
$$

Hence, the argument above is still valid, as we can trade $\exp (X(t))$ apporoximately by entering a roll-over position in the available forward contracts with the longest time until settlement. We will make use of this fact in our empirical analysis of Fish Pool prices in the next section. 

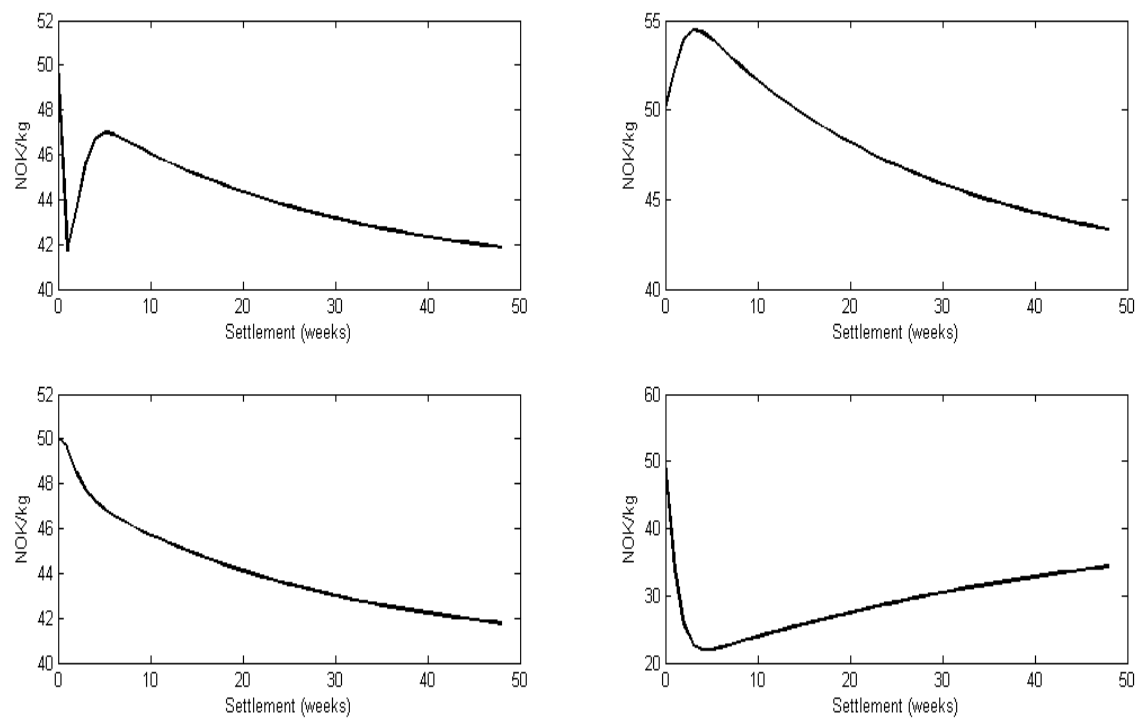

FIGURE 2. Plot of some simulated example forward curves.

In Fig. 2 we show four different shapes that we can achieve using our stochastic model. We have computed the forward curves based on a CAR(3) model (i.e., letting $p=3$ foir $\mathbf{Z}$ ), and a non-stationary factor being a driftless (i.e., $\mu=0$ ) Brownian motion with volatility $\eta=0.05$. We assumed the parameters in the $A$-matrix to be $\alpha_{3}=0.04, \alpha_{2}=1.331$ and 2.124 , volatility $\sigma=0.0671$ and long term level $\xi=3.625$, parameters which happen to coincide with the estimated ones as we will see in the next section. Furthermore, we assumed $X(0)=0$ and $Z_{1}(0)=\log (50)$, which means that the current price of salmon is set to $50 \mathrm{NOK} / \mathrm{kg}$ if $Z_{2}(0)=Z_{3}(0)=0$. . In the figure, we plot the choices (clockwise) $Z_{2}(0)=$ $-0.5, Z_{3}(0)=1, Z_{2}(0)=0.02, Z_{3}(0)=0.1, Z_{2}(0)=-0.3, Z_{3}(0)=-0.5$ and $Z_{2}(0)=0.02, Z_{3}(0)=$ -0.1 . These various choices specify the slope and curvature of the "current" salmon price. Thus, in the upper left hand corner we see a negative slope with positive curvature, leading to an interesting doublehump shape similar to the one we observe in Fig. 1 for Aug 12, 2013. The lower left hand corner shows the shape when the curvature is negative, and we have a very different term structure. The two other plots depict positive slope, but positive and negative curvature, and we obtain similar term structures as in Fig. 1 as ub April26, 2011.

\section{DATA DESCRIPTION AND EMPIRICAL ANALYSIS}

In this Section we estimate our proposed stochastic model for the spot and foward prices on salmon to observed data. The empirical analysis is performed on a data set of FPI index quotes and forward prices obtained from the Fish Pool. ${ }^{2}$

The Fish Pool organizes trade in salmon forward contracts that are settled on the FPI index. As earlier mentioned, the settlement takes place as an average of the FPI index over a monthly period. Hence, for trading each day there are a number of forward contracts quoted with various monthly settlement periods. ${ }^{3}$

We had available a set of 60 monthly forward prices for each trading day ranging from 5 August 2009 untill 18 July 2016, altogether 1797 trading days. The FPI index is quoted every Tuesday, and we had accessible FPI index data from week 33 in 2009 until week 23 in 2016, altogether 356 observations. The forward prices and the FPI index are both denominated in NOK/kg.

\footnotetext{
${ }^{2}$ Forward prices were downloaded from http://fishpool.eu/price-information/forward-prices-3/forward-closing-prices-history/, while the FPI index from http://fishpool.eu/price-information/spot-prices/history/

${ }^{3}$ In the market, Fish Pool offers other settlement periods as well, such as quarters and years. These are simple aggregation of monthly settled contracts.
} 


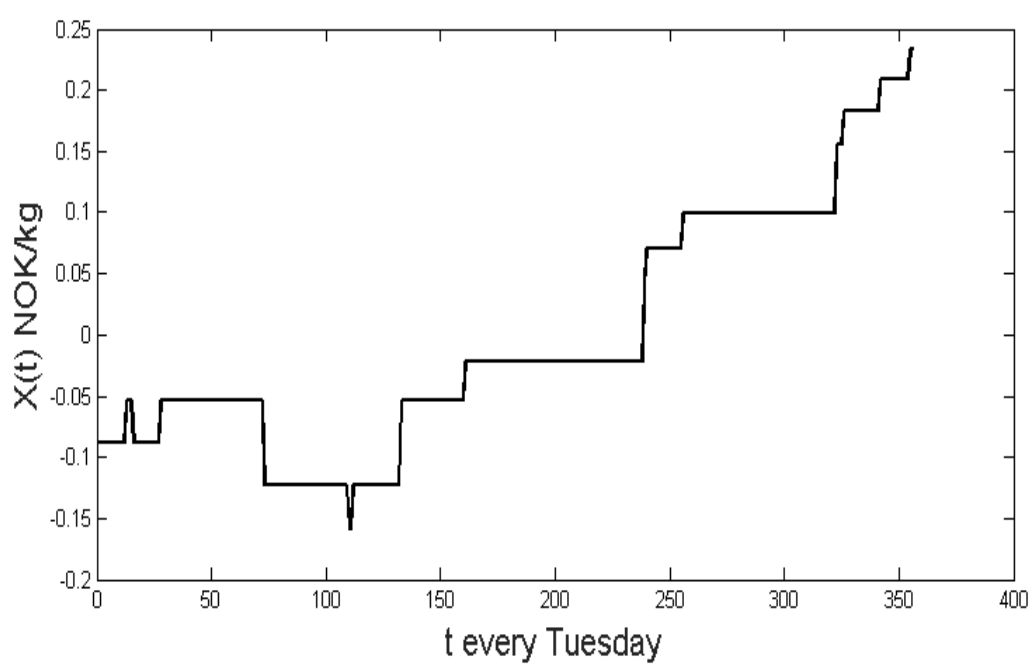

FIGURE 3. Plot of the filtered time series of $X$.

In estimating the parameters of our FPI model, we do the following: First we filter out the long term factor $X$ from forward prices with long time until settlement. This gives us a time series $x_{1}, x_{2}, \ldots, x_{N}$ of $X(t)$, for each trading day in the sample. Next, we use these observations to get data for the stationary component $Y$ in the FPI. As the FPI is only observed on Tuesdays, we single out the $x_{i}^{\prime} s$ which are observed on Tuesday, labelled $x_{j}, j=1, \ldots, M$, and derive

$$
y_{j}=\ln s_{j}-x_{j}
$$

for $j=1, \ldots, M$, with $s_{j}$ being the $j$ th observation of the FPI available in our data set. This provide us with a data set for $X$ and $Y$, on which we perform a time series analysis. Conveniently, we let time $t$ be measured in weeks here, i.e., $t=1$ corresponds to one week.

In the first step, where we filter out $X$, we make use of Prop. 3 with the choice of $\theta_{L}$ given in (8). We selected the contract which is farthest out on the forward curve (which is the 60th contracts in our data set) to have prices data $F\left(t, T_{1}, T_{2}\right)$ with $T_{1}>>t$. We remark in passing that on a few occasions (7, in fact) the forward prices for the 60th contract were zero, in which case we used the 59th contract. Indeed, as the observed forward curves are flat in the long end, one can substitute with other forward contracts which are closer in maturity without altering the result. We estimated the long-term level $c$ to be,

$$
\widehat{c}=3.4554 \text {. }
$$

This estimate is based on the mean value of the logarithmic forward prices farthest out on the curve. In Figure 3 we see the time series of the filtered $X$. The stepwise path strongly points towards a compound Poisson process as a suitable model for $X$, i.e.,

$$
X(t)=L(t)=\sum_{k=1}^{N(t)} J_{k}
$$

where $N$ is a Poisson process with jump intensity $\lambda$ and $\left\{J_{k}\right\}_{k \in \mathbb{N}}$ being iid random variables. We observe altogether 15 jumps in the path of $X$, which means that we have a jump intensity of $\widehat{\lambda}=0.042$ (on a weekly time scale). To fully specify the model for $X$ we need a distribution for the random variables $J_{k}$. Based on only 15 observations, this may prove difficult. As we see from Figure 3, we have predominantly positive jumps, but we also observe jumps downwards. A positively skewed distribution seems appropriate. One may specify this distribution further including some market knowledge about the future, or simply using the small number of data in order to estimate it. In Figure 4 we plot the histogram of the 15 jumps with 5 bins.

After subtracting the sub-sample of $x_{j}$ 's observed on Tuesdays, we obtain a sample $y_{1}, \ldots, y_{M}$ of data for the short-term factor $Y$. In our case, $M=356$, and we show the time series of data in Figure 5 . 


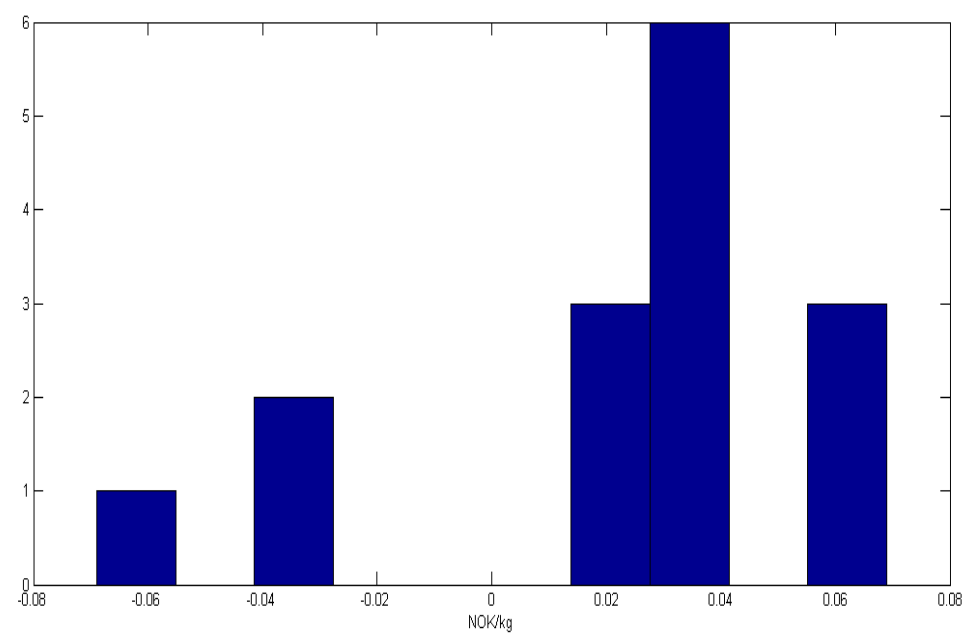

FIGURE 4. Histogram of the observed jumps of $X$.

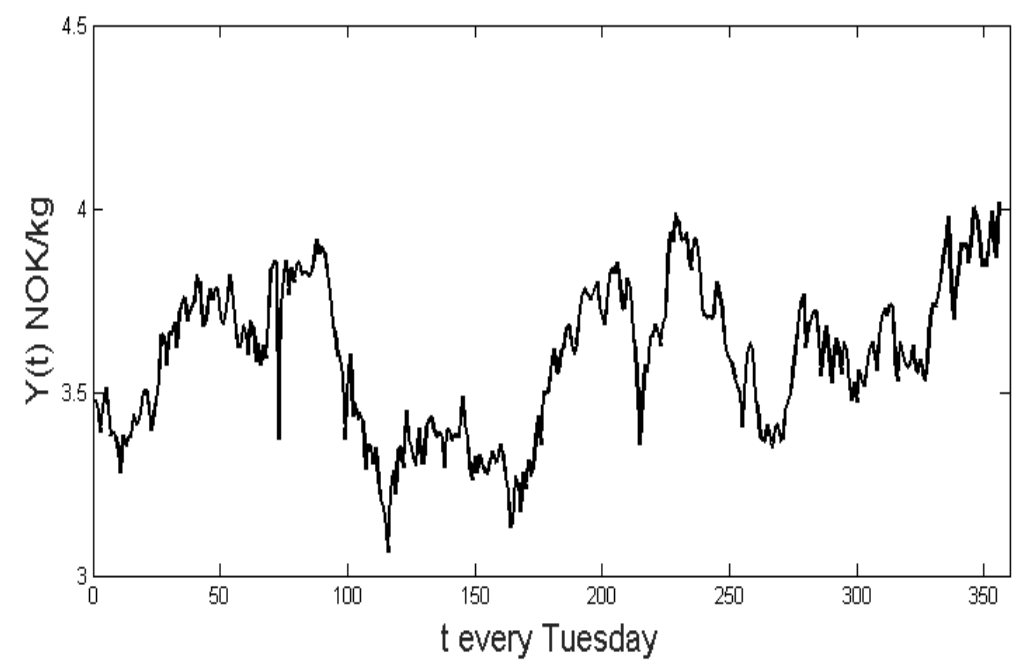

FIGURE 5. The time series of observations of $Y$.

Motivated by the autocorrelation function (ACF) and the partial ACF of the time series (see Figure 6), we propose an autoregressive model of order 3, i.e., an AR(3) dynamics of the time series. Using R, we estimated the following,

$$
y_{t}=0.145+0.876 y_{t-1}-0.083 y_{t-2}+0.167 y_{t-3}+\sigma \epsilon_{t},
$$

for $\epsilon_{t}$ being IID normal noise. The estimate of the standard deviation of the residuals is $\widehat{\sigma}=0.0671$. The corresponding CAR(3)-model can be identified by appealing to Example 10.2 in Benth et al. [3], giving an $A$-matrix with elements $\widehat{\alpha}_{1}=2.124, \widehat{\alpha}_{2}=1.331$ and $\widehat{\alpha}_{3}=0.040$. The eigenvalues of $A$ become $\lambda_{1}=-0.0316$ and $\lambda_{2,3}=-1.0462 \pm 0.4127 \mathrm{i}$, all having negative real part as desired. Hence, the fitted CAR(3)-process $Y$ is stationary with a Gaussian limiting distribution. Suppose $\boldsymbol{\xi}=(\xi, 0,0)^{\top}$, for $\xi \in \mathbb{R}$. Performing an Euler discretization of the model of the CAR(3)-model with level $\boldsymbol{\xi}$, following the scheme as in Section 10.2 of Benth et al. [3], yields that the time series model of $Y$ will have the constant term $\alpha_{3} \xi$. Hence, $\widehat{\xi}=3.625$. 

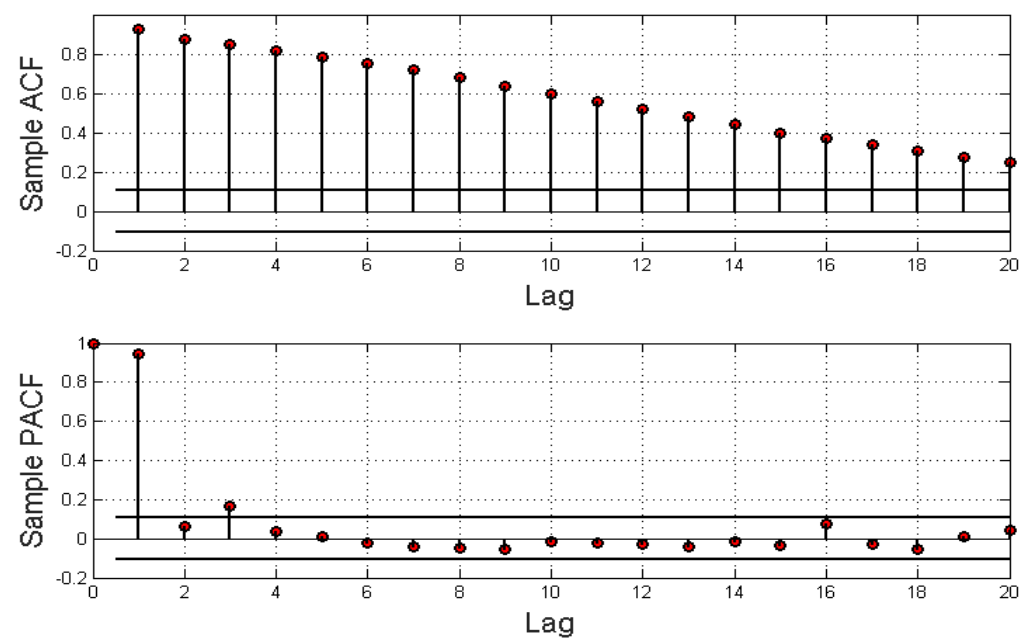

FIgURE 6. The ACF and partial ACF $Y$. The solid horizontal lines mark the 95\% confidence band

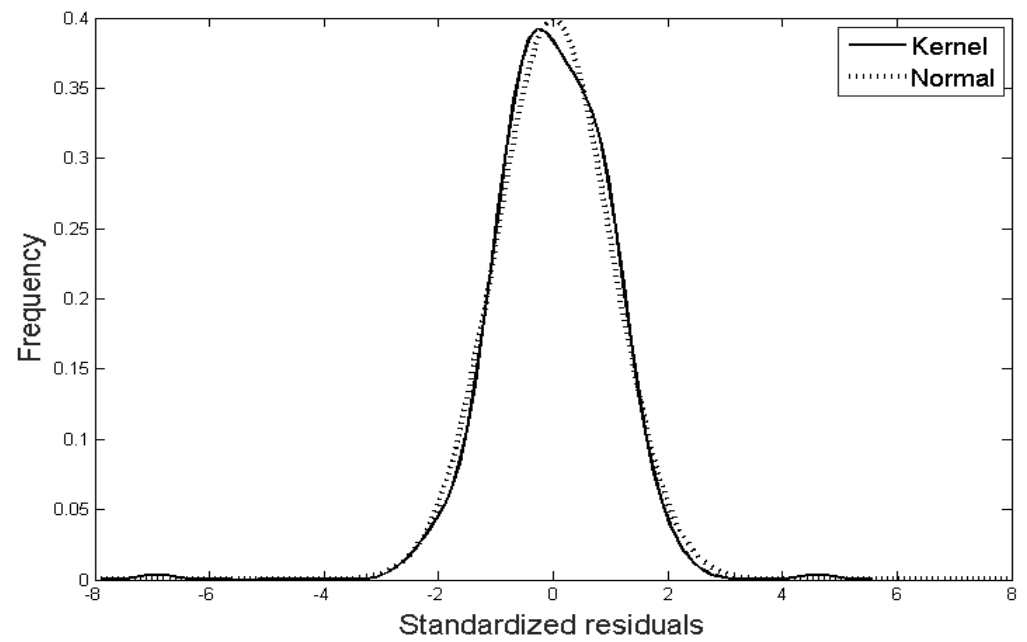

FIGURE 7. The fitted normal distribution (dotted) and the empirical distribution (solid) of the residuals of the AR(3) model

Remark 4. Notice that we can also estimate $\boldsymbol{\xi}$ from the definition of $c$ in Prop. 3 and the estimate in (10). From the definition of $c$ we find that the first coordinate of $\boldsymbol{\xi}$ can be estimated by

$$
\widehat{\xi}=\widehat{c}-\frac{\widehat{\sigma}^{2}}{2} \int_{0}^{\infty}\left(\mathbf{e}_{1}^{\top} e^{A u} \mathbf{e}_{3}\right)^{2} d u .
$$

Using this estimator of $\xi$, we get $\widehat{\xi}=3.434$. Although different, the estimated $\xi$ is not too much deviating from our estimate above.

We finalize the empirical estimation with validating the normality assumption of the residuals $\epsilon_{t}$ in the AR(3) time series. Indeed, in Figure 7 we see that the empirical density of the residuals (solid is reasonably close to the fitted (standard) normal distribution (dotted). A kernel smoother in Matlab was used to produce the empirical density. We also looked at the ACF of the residuals, which were not significantly different than zero for all lags (based on a Box-Ljung test with 95\% confidence level). The ACF of the squared 
residuals, however, showed some signs of non-zero correlation for small lags, giving an indication of nonindependence in the data and possibly stochastic volatility effects. We will not puruse this further here, but using the above results as a motivation for modelling the residuals by white noise, and thereby giving a rationale for the Brownian assumption in the CAR(3) dynamics of $Y$.

Thus, we have fully specified the dynamics of $X$ and $Y$. To obtain a full specification of the forward price dynamics, we still need to assess the value of the market price of risk $\theta_{B}$. An empirical analysis of the risk premium in the salmon forward market is the focus in the next Section.

\section{EMPIRICAL STUDY OF THE RISK PREMIUM}

The risk premium is defined to be the difference between the actual forward price and predicted spot for a given settlement period. If the settlement period is $\left[T_{1}, T_{2}\right]$, the predicted price is defined as

$$
F_{P}\left(t, T_{1}, T_{2}\right)=\frac{1}{T_{2}-T_{1}} \int_{T_{1}}^{T_{2}} \mathbb{E}\left[S(T) \mid \mathcal{F}_{t}\right] d T .
$$

Our theoretical forward price is recovered using the expectation with respect to $Q$ rather than $P$ inside the integral above. We find from Prop. 7 that

$$
f_{0}(t, T):=\exp \left(h(T-t)+X(t)+\mathbf{e}_{1}^{\top} e^{A(T-t)} \mathbf{Z}(t)\right)
$$

with

$$
h(x)=\mathbf{e}_{1}^{\top}\left(I-e^{A x}\right) \boldsymbol{\xi}+\frac{\sigma^{2}}{2} \int_{0}^{x}\left(\mathbf{e}_{1}^{\top} e^{A u} \mathbf{e}_{3}\right)^{2} d u
$$

for $x \geq 0$. Notice that we have fixed $\psi_{Q}(-\mathrm{i})=0$ according to (8), so when we consider the predicted spot, we in reality put $\theta_{B}=0$. The risk premium is thus,

$$
R\left(t, T_{1}, T_{2}\right):=F\left(t, T_{1}, T_{2}\right)-\frac{1}{T_{2}-T_{1}} \int_{T_{1}}^{T_{2}} f_{0}(t, T) d T,
$$

with $F\left(t, T_{1}, T_{2}\right)$ the observed forward price at time $t$ for a contract settled over $\left[T_{1}, T_{2}\right]$.

To avoid cumbersome numerical integration of $f_{0}(t, T)$, we use the middle point in the settlement period as an approximation. That is, we use the approximation

$$
\frac{1}{T_{2}-T_{1}} \int_{T_{1}}^{T_{2}} f_{0}(t, T) d T \approx f_{0}\left(t, T_{1,2}^{*}\right)
$$

where $T_{1,2}^{*}:=\left(T_{1}+T_{2}\right) / 2$. To derive the risk premium, we compute $f_{0}\left(t, T_{1,2}^{*}\right.$ based on observations of $X(t)$ and $\mathbf{Z}(t)$, and the value of $h(T-t)$ using the estimated parameters for $A, \xi$ and $\sigma$. In computing the function $h$, we do a simple Riemann approximation of the integral. Finally, we recall from the definition of $\mathbf{Z}$ that $Z_{1}^{\prime}(t)=Z_{2}(t)$ and $Z_{2}^{\prime}(t)=Z_{3}(t)$, or, $Z_{1}^{\prime \prime}(t)=Z_{3}(t)$. Therefore, we can recover the coordinates $Z_{2}(t)$ and $Z_{3}(t)$ in $\mathbf{Z}(t)$ from the numerical derivatives of $Z_{1}(t)$, with $Z_{1}(t)$ actually being $Y(t)$.

The FPI is only available every Tuesday, and although the market trades other weekdays, we focus here only on the Tuesday forward prices in our study of the risk premium. Furthermore, the numerical derivatives of $Y(t)$ is $Y^{\prime}(t) \approx Y(t)-Y(t-1)$ and $Y^{\prime \prime}(t) \approx Y(t)-2 Y(t-1)+Y(t-2)$, with $t$ being measured on a weekly time scale. These approximations are rather crude, but provides a simple alternative to filtering techniques to include observed data of the FPI and $X$ when assessing the risk premium.

In Fig. 8 we plot the resulting risk premium as a function of time and time to settlement. From the figure we reach the overall conclusion that the risk premium in the long end of the forward market is negative, which is in line with the classical theory of backwardation in commodity markets (see Geman [16] and references therein). In the short end of the forward market, however, we find that the risk premium can be both negative and positive. Thus, it seems that producers are putting on a hedging pressure in the long end of the forward market, while in the short end the consumers may also be dominating in hedging activities over the producers. Indeed, the situation seems similar to that of power markets, where Geman and Vasicek [18] argued for retailers' need to hedge spike risk, leading to occasional positive risk premia in the short end of the power forward market (see Benth, Cartea and Kiesel [4] and Bessembinder and Lemon [7] for more theoretical and empirical analysis of this fact in power markets). One may speculate that consumers hedge the spot price risk in the salmon market using contracts which are close in settlement, including exporters tied up in fixed-price contracts of delivery of salmon. 


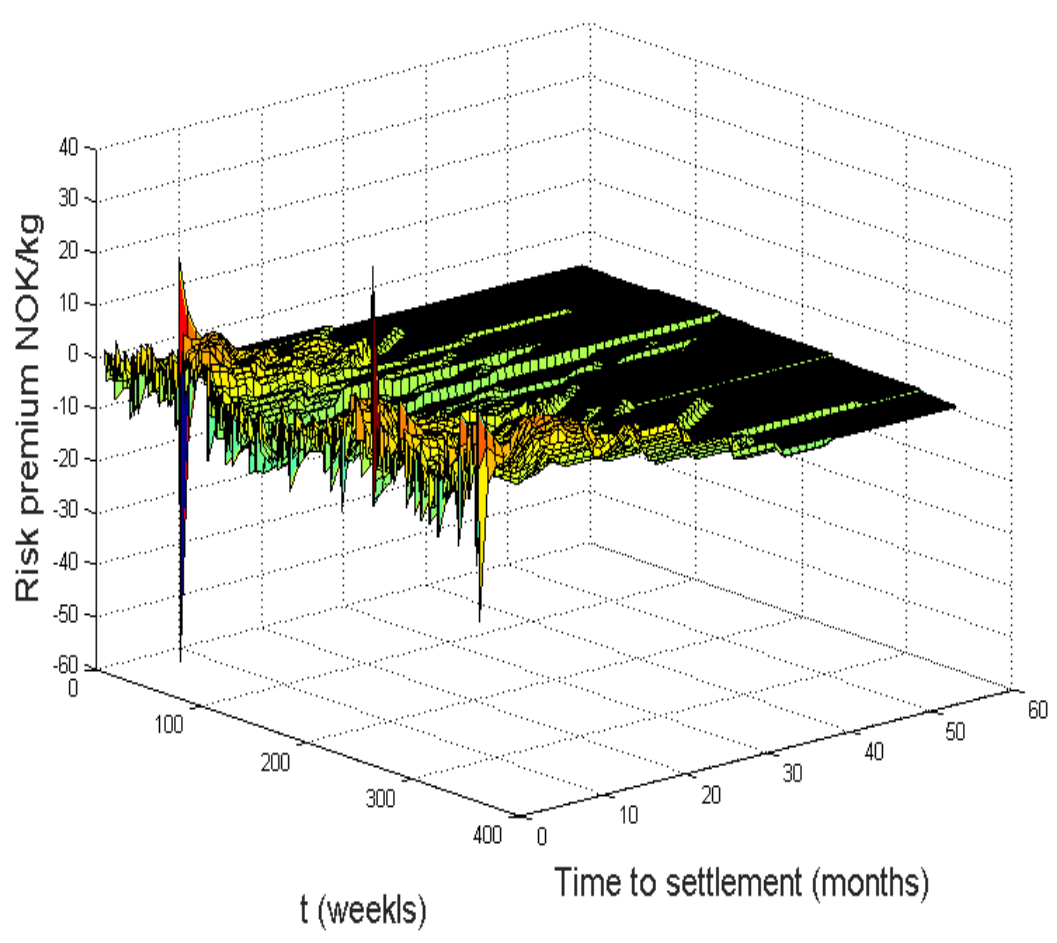

FIGURE 8. The risk premium as a function of time (in weeks) and time to settlement (in months)

We notice the stochastic behaviour of the risk premium, especially in the short end of the market. This points towards a state-dependent pricing measure $Q$, as proposed in Benth and Ortiz-Latorre [5] for Lévy-driven Ornstein-Uhlenbeck processes and in Benth and Šaltytè Benth [2] for CARMA-processes with Brownian noise. For an Ornstien-Uhlenbeck dynamics, Benth and Ortiz-Latorre [5] propose a measure change from $P$ to $Q$ for which the speed of mean reversion is slowed down. This measure change will have a Radon-Nikodym derivative which is explicitly depending on the state of the Ornstein-Uhlembeck process, that is, on the spot price dynamics. As a consequence of this, the risk premium will explicitly depend on the underlying spot dynamics and thus stochastically varying. This is very different from the more classical Esscher transform approach with constant market price of risk to construct pricing measures in commodity markets, where the risk premium will become deterministic (see Benth, Šaltyte Benth and Koekebakker [3] for more on the Esscher transform in energy and related markets). Benth and Ortiz-Latorre [6] perform an empirical study of the temperature forward market using the state-dependent change of measure for CARMA processes in Benth and Šaltyte Benth [2]. The context is similar for the salmon market, where one faces a settlement period and a memory in the underlying spot (e.g. FPI) dynamics.

We show the risk premium histogram for front month (top left), 15 (top right), 25 (bottom left) and 35 (bottom right) months to maturity in Fig. 9 The risk premia are gradually shifting from a rather symmetric distribution around zero to a more skewed distribution on the negative half-line when moving from the short to the long end of the forward curve. For the front month, the risk premium is roughly concentrated between $\pm 10 \mathrm{NOK} / \mathrm{kg}$, while in the very long end it is between $-4 \mathrm{NOK} / \mathrm{kr}$ to $-10 \mathrm{NOK} / \mathrm{kg}$. These particular cases of forward risk premia confirm our more general conclusions above.

Our empirical findinings on the risk premium strongly suggest that a constant market price of risk $\theta_{B}$ at best can only describe the average of the risk premium and not its stochastic variations. Recalling that the forward prices are quoted daily with an underlying index only revealed once a week, it would be a challenging task to combine a state-dependent pricing measure $Q$ with filtering techniques on the FPI to obtain daily assessments of it. This is left for future studies. 

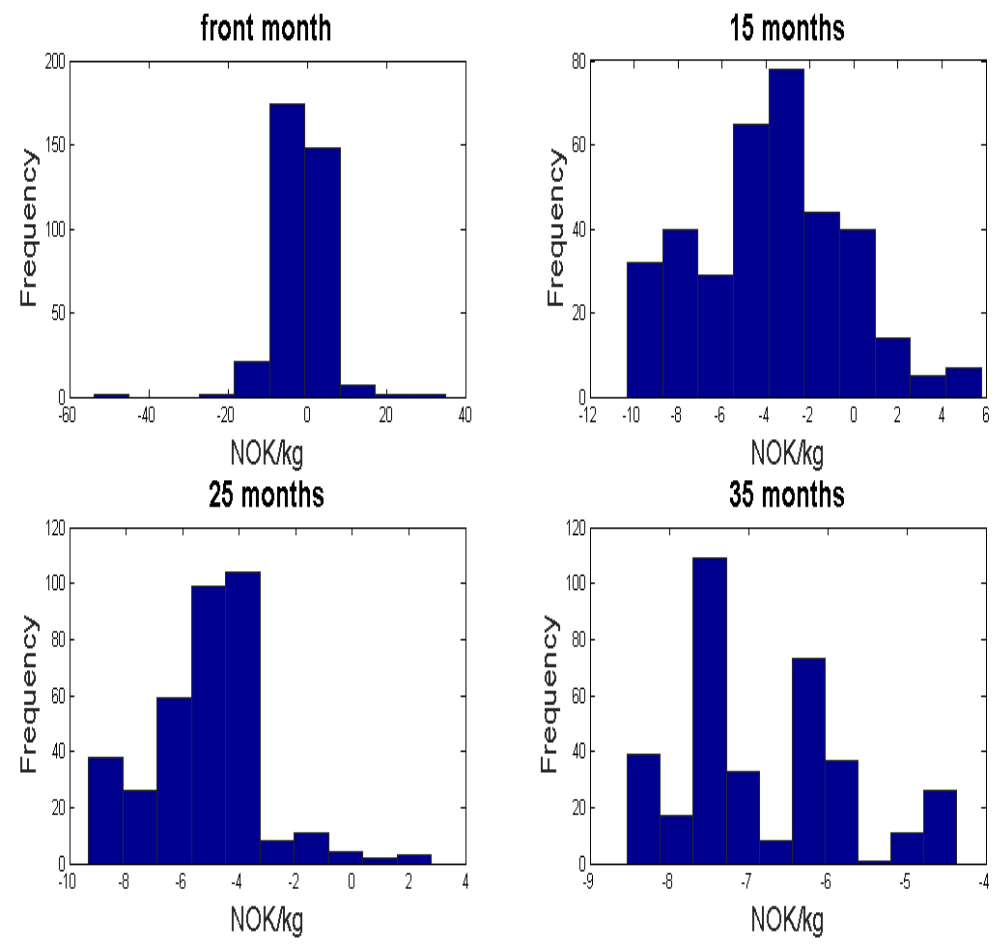

FIGURE 9. The histogram of risk premia for a selection of settlement times, with the front month (top left), 15 (top right), 25 (bottom left) and 35 (bottom right) months to maturity

\section{CONCLUSION}

All species adapt to their surrounding environments, and farmed salmon living in the ocean may be affected by climate change. Furthermore, climate change may have a severe impact on the world economy (Dietz et al. [11]). Our results highlight an opportunity for future research on the underlying assumptions in financial models to understand if and how changes in climate and biodiversity may affect the financial markets (Reeves et al. [21], Dalton [10], Galaz et al. [15]). In this paper we present a two-factor model for the underlying spot, a CARMA and non-stationary CPP that show a stochastic risk premium with changing sign in short end, similar to what has been observed for power markets. Hence, our results indicate that also in the fish market forwards may be used as a protection against price risk by both producers and consumers. Like all models, our model also has limitations, in particular the fact that the data are being quoted only Tuesdays. This causes a filtering procedure by market participants trying to find the hidden FPI index in remaining days when quoting forward prices. More analysis of the long-term non-stationary factor is needed; but currently, there is a sparse amount of data available In future studies we also want to include a state-dependent pricing measure, that can change the coefficients in the CAR-matrix $A$, corresponding to a change in the speed of mean reversion (see Benth and Šaltytė Benth [2]). This could provide a better description to the observed risk premium in the market.

\section{REFERENCES}

[1] F. Asche, R. E. Dahl, and M. Steen (2015). Price volatility in seafood markets: farmed vs. wild fish. Aquaculture Economics \& Management, 19(3), pp. 316-335.

[2] F. E. Benth and J. Šaltytė Benth (2013). Modeling and Pricing in Financial Markets for Weather Derivatives. World Scientific.

[3] F. E. Benth, J. Šaltytė Benth and S. Koekebakker (2008). Stochastic Modelling of Electricity and Related Markets. World Scientific.

[4] F. E. Benth, A. Cartea and R. Kiesel (2008). Pricing forward contracts in power markets by the certainty equivalence principle. J. Banking \& Finance, 32(10), pp. 2006-2021. 
[5] F. E. Benth and S. Ortiz-Latorre (2014). A pricing measure to explain the risk premium in power markets. SIAM J. Financial Math., 5, pp. 685-728.

[6] F. E. Benth and S. Ortiz-Latorre (2017). Calibration of temperature futures by changing the mean reversion. J. Energy Markets, 10(1), pp. 1-25.

[7] H. Bessembinder and M. L. Lemon (2002). Equilibrium pricing and optimal hedging in electricity forward markets. J. Finance, 57, pp. 1347-1382.

[8] N. Boston (2012). Trading the oceans: the brave new world of seafood futures contracts. 18 Ocean \& Coastal L.J. 285, 20122013.

[9] P. J. Brockwell (2001). Lévy-driven CARMA processes. Ann. Inst. Stat. Math., 53, pp. 113-124.

[10] R. Dalton (2005). Conservation policy: Fishy futures. Nature, 437, pp. 473-474.

[11] S. Dietz, A. Bowen, C. Dixon, and P. Gradwell (2016). Climate value at risk of global financial assets. Nature Clim. Change, advance online publication

[12] D. Duffie (1992). Dynamic Asset Pricing Theory. Princeton University Press.

[13] Ewald, C-O. (2013). Derivatives on nonstorable renewable resources: fish futures and options, not so fishy after all. Natural resource modeling, 26(2), 215-236.

[14] C.-O. Ewald and P. Salehi (2015). Salmon futures and the Fish Pool Market in the context of the CAPM and the Fama \& French three-factor model. Available at SSRN: https://ssrn.com/abstract=2567737

[15] V. Galaz, J. Gars, F. Moberg, B. Nykvist, and C. Repinski (2015). Why ecologists should care about financial markets. Trends Ecol. Evol., 30, pp. 571-580.

[16] H. Geman (2005). Commodities and Commodity Derivatives. Wiley-Finance, John Wiley \& Sons.

[17] R. Gibson and E. S. Schwartz (1990). Stochastic convenience yield and the pricing of oil contingent claims. J. Finance, 45(3), pp. 959-976.

[18] H. Geman and O. Vasicek (2001). Forwards and futures on non-storable commodities: the case of electricity. RISK, August issue.

[19] I. Karatzas and S. E. Shreve (1991). Brownian Motion and Stochastic Calculus. Springer Verlag.

[20] J. Lucia and E. S. Schwartz (2002). Electricity prices and power derivatives: evidence from the Nordic Power Exchange. Rev. Derivatives Res., 5(1), pp. 5-50.

[21] M. Reeves, K. Haanaes, C. Love and S. A. Levin (2012). Sustainability as adaptability. J. Applied Corporate Finance, 24, pp. 14-23.

Fred Espen Benth, University of Oslo, Department of Mathematics, P.O. Box 1053, Blindern, N-0316 OSLO, NORWAY

E-mail address: fredb@math.uio.no

Anne Maria Eikeset, University of Oslo, Centre for Ecological and Evolutionary Synthesis (CEES), N-0316 Oslo, Norway, AND, CENTre For BioComplexity, Princeton University, NJ 08544, AND, Princeton ENVIronmental Institute, Princeton University, NJ 08544, and, Department of Ecology and Evolutionary BiolOGY, PRINCETON UNIVERSITY, NJ 08544

E-mail address: a.m.eikeset@ibv.uio.no

Simon Asher Levin, Centre for BioComplexity, Princeton University, Nu 08544, And, Princeton Environmental Institute, Princeton University, NJ 08544, AND, Department of ECOlOGy and Evolutionary Biology, PRINCETON UNIVERSITY, NJ 08544

E-mail address: slevineprinceton.edu

Wanjuan Ren, University of Oslo, Department of Mathematics, P.O.Box 1053 Blindern, N-0316 Oslo, NORWAY

E-mail address: wanjuanr@math.uio.no 